\title{
Quality Standards Maintenance using Electronic Sensors in Dairy Products
}

\author{
Priya M. \\ Dept of ECE, \\ Sambhram Institute \\ of Technology, \\ Bengaluru
}

\author{
Namratha S. \\ Dept of ECE \\ Sambhram Institute \\ of Technology, \\ Bengaluru
}

\author{
Asha R.K. \\ Dept of ECE, \\ Sambhram Institute \\ of Technology, \\ Bengaluru
}

\author{
Bhuvaneshwari \\ $M$. \\ Dept of ECE, \\ Sambhram Institute \\ of Technology, \\ Bengaluru
}

\author{
K. Ezhilarasan \\ Dept of ECE, \\ Sambhram Institute \\ of Technology, \\ Bengaluru
}

\begin{abstract}
Milk is one of the best sources of immunity. It is consumed by people of all ages. It gives immunity to fight against various diseases. However, a few people deliberately add particular substances to milk to decrease its quality and thereby adulterate it. These substances are called as defilements. The adulteration of milk implies that the quantity of milk is being increased by adding some common adulterants such as flour, detergents etc thereby leading to compromise in quality. These adulterants can cause damage to the organs of our body. The immediate effect of drinking adulterated milk with urea, caustic soda and formalin is gastroenteritis and the longterm effects are known to be far more serious. Therefore in order to get rid of these problems or at least reduce them to a significant level, newer schemes need to be implemented by bringing in sensor based techniques in the field of detection of adulteration system. Our aim is to identify certain adulterants present in milk and its products such as flour, detergents, formaldehyde, acidic substances such as urea with the help of smart electronic sensors.
\end{abstract}

\section{Keywords}

Milk, quality of milk, adulterants, sensors, ph, pollutants

\section{INTRODUCTION}

Milk is a nutritive drink which contains all the fundamental segments of like proteins, fats, vitamins and minerals. On dry basis, raw whole milk contains $29.36 \%$ fat, $26.98 \%$ protein ( $22.22 \%$ casein, $4.76 \%$ whey proteins), $38.1 \%$ lactose, and $5.56 \%$ ash. The composition of non- fat solids of skim milk is: $52.15 \%$ lactose, $38.71 \%$ protein $(31.18 \%$ casein, $7.53 \%$ whey protein), $1.08 \%$ fat, and $8.06 \%$ ash. The $\mathrm{pH}$ of normal milk varies from 6.4 to 6.8 . As indicated by the Food and Agriculture Organization of the United Nations, the utilization of milk and milk items is by more than 6 billion individuals around the world. In India, Dairy farming can be seen to a great extent. In order to gain more finance, the merchants while packing the fresh, pure milk in the packets add adulterants and try to increase the quantity. This milk is then sold in market and is consumed by people. Along with milk, even the milk products are adulterated to a great extent. Khoa is one of the most polluted milk products. The immediate effect of drinking adulterated milk with urea, caustic soda and formalin is gastroenteritis and the long-term effects are known to be far more serious.

\section{LITERATURE SURVEY}

Saadia H.H Shinawy, AdelM.EL-Kholy and et.al in [1] give detailed information about materials and methods that can be used and their results. The major problem that authors have discussed here is that the cow's milk samples were subjected to adulteration by addition of water than buffalo's milk samples as it is low in fat content and any partial removal of fat can be identified. The solutions mentioned here are for some laboratory examined samples which cannot be detected by common people. In our project/study we are trying to overcome this by constructing a device that is easy to use and available to common man also.

ManjotKaur and JiwanjotKaur in [2] mentioned about the various dairy products and their major adulterants. This paper gives the information about limited products where as in our project adulteration can be detected in few more products.

Dr.G.Rajakumar et.al [3] have aimed to present some aspects regarding milk quality and quantity estimation. . High quality milk must have no salinity, so salinity of the milk is measured by using a salinity sensor for detecting adulteration of milk and level of the milk will be measured by using a level sensor for measuring the quantity of the milk. When the milk is stored for long, the microbial activity gets started which gives the milk a foul smell which can be detected using a gas sensor. But this system cannot detect the presence of steroids in milk. It has not been indicated whether this process can also be used for milk products.

Jai Desai et.al [4] have aimed to provide the adulteration technique which is non-contact type method. This can solve the problem of relying on the traditional methods which involves a lot of wastage of sample during the detection of adulteration. Optical sensors which were used provide good results but this does not include the fat content in the milk sample. Fat content changes the milk density which affects the refractive index of the milk. Cow's milk has less fat content than buffalo's milk.

DadasahebNavale et.al [5] have given the detailed information of detection of an adulteration present in milk and milk products in which many tests are conducted to find the presence of water, starch, urea, detergent, glucose and also to detect gelatin in cream \& presence of sucrose in khoa. This paper indicates methods to detect various types of adulteration present in milk and milk products.

Tanzina Azad et.al [6] presents a detailed review of common milk adulterants as well as different methods to detect the adulterants both quantitatively and qualitatively. This study is organized to be an adulterant based study instead of techniques based one. Apart from regular techniques, recent development in these detection techniques has also been reported. This review intends to contribute towards the common knowledge base regarding possible milk adulterants and their detection techniques.

Lee et.al [7]. sought to improve upon the methylene blue reduction method while maintaining its advantages by 
supplementing it with an amperometric sensor. An amperometric sensor composed of a circuit with a potentiostat and a pair of electrodes, measures current change. Amperometric sensors are small and inexpensive and have been tested in a variety of media to detect changes in bacteria such as E.coli. Lee et al. inoculated with milk E. coli and ENT. Aerogenes are two types of coli forms that indicate the sanitary condition. However, this method cannot discriminate between viable and non-viable cells. Furthermore, type of bacteria detection was lacking. The amperometric sensor could only detect E. coli and Ent. Aerogenes coliforms when other bacteria such as B. subtilis, Lactobacillus sp., Saccharomyces sp., and Staph. Aureus were tested upon; they produced a negligible current change

Al-Qadiri, M.Lin, Al-Holy et.al [8]. mentioned that they evaluated visible and short wavelength near-infrared diffuse reflectance spectroscopy (SW-NIR) as a technique to detect milk spoilage in pasteurized skim milk. They wanted to see the feasibility of applying visible and SW-NIR spectroscopy to monitor spoilage of pasteurized skim milk in industrial settings. In doing so, Al-Qadiri et.al first took the total aerobic plate count and $\mathrm{pH}$ measurements. The mean $\mathrm{pH}$ measurement for control milk samples was 6.66, and they found no obvious $\mathrm{pH}$ decrease for milk samples stored at $6^{\circ} \mathrm{C}$ after 30 hours of storage. In experimental samples, the visible and SW-NIR diffuse spectroscopy detected the formation of metabolic byproducts from proteolysis and lipolysis caused by bacterial cell growth, which led to a reduction in $\mathrm{pH}$. This method was effective, but costly. So here in our project we are planning to implement a cost effective method for the same

Nicolaou et.al [9]. attempted to take infrared spectroscopy further with matrix-assisted laser desorption/ionization timeof-flight mass spectrometry has already been used in protein and peptide identification and quantification; however, Nicolaou et.al wanted to see if it was useful for microbial spoilage assessment because techniques for identifying and quantifying spoilage bacteria in pasteurized milk are timeconsuming. Their methodology included incubating milk samples and raw pork meat samples at $15^{\circ} \mathrm{C}$ and at room temperature, and then analyzing them at a rate of 4- minute intervals. It's most comparable technology is Fourier transform infrared (FT-IR) spectroscopy. However, MS allows more equivocal identification of important proteins while FT-IR spectroscopy does not, or does so at best only empirically through peak assignments. Drawbacks, however, include the limited use of infrared spectroscopy in the field. The technology is perceived as a tool for assessing protein qualitatively rather than for measuring microbial bacterial count quantitatively. Familiarization can help change perceptions and lead to use of this technology in the dairy industry. However, the technical difficulty of this method renders it unsuitable for consumer use.

\section{PROPOSED SYSTEM}

The proposed system can detect the pollutants present in milk and its products using e-technique and ensures that the quality of the milk is good for both the sellers as well as the consumers. This project can be used to provide simple and rapid test procedures with a lesser time line. The method of detecting pollutants in source level may also help to protect cows from getting affected due to injection of hormones. By this project we can also ensure that the consumers don't end up paying more for the substandard milk and hence control corruption. It consists of interconnection of Arduino board, $\mathrm{pH}$ sensor, temperature sensor, viscosity sensor, battery, gas sensor, formaldehyde sensor, LCD, LED. The quality of milk can be administrated with the help of the smart sensors employed. If there is any adulteration, the same can be indicated using LCD display and LEDs.

PH Sensor: This sensor is used to determine changes in the $\mathrm{pH}$ of milk, generally the $\mathrm{PH}$ of the milk lies between 6.4 to 6.8. If the $\mathrm{pH}$ is less than 6.4 and greater than 6.8 then milk is acidic or alkaline respectively. $\mathrm{pH}$ sensor helps to measure the acidity or alkalinity of the water with a value between $0-14$. When the $\mathrm{pH}$ value dips below seven, the water starts to become more acidic. Any number above seven equates to more alkaline. Each type of $\mathrm{pH}$ sensor works differently to measure the quality of the water.

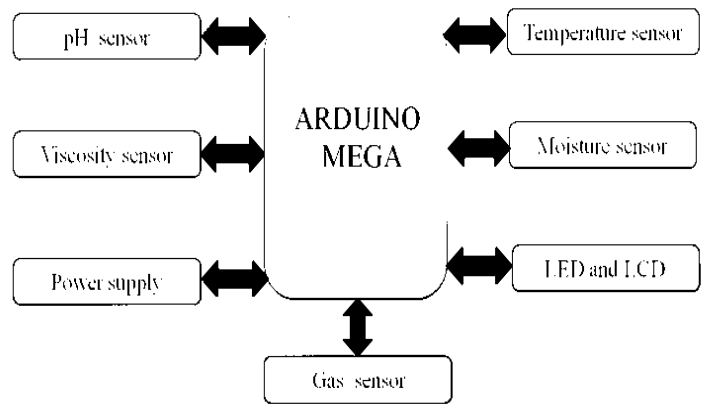

Fig 1: Block diagram for detection of adulterants in milk

Viscosity Sensor: It enables continuous monitoring of viscosity, relative dielectric number and temperature of lubricating and hydraulic oils in order to provide effective real-time assessment of the oil condition. It is used to determine whether the viscosity of milk is affected. Viscosity of milk can be increased by adding flour, detergents and decreased by adding water. By measuring the rate of flow of particles, we can get an idea whether the milk is being adulterated or not

Liquid Temperature Sensor: DS18B20 is the liquid temperature sensor. A temperature sensor is an electronic device that measures the temperature of its environment and converts the input data into electronic data to record, monitor, or signal temperature changes. Milk has its own temperature criteria it should be maintained during storage. Generally milk will be safe at the temperature range of 35 to $40 \mathrm{degF}$, above or below which the formation of bacteria occurs and does not fit for consumption.

Moisture Sensor: Moisture sensor is a simple breakout for measuring the moisture in starch and similar materials. The two large exposed pads function as probes for the sensor, together acting as a variable resister. One commonly known issue with moisture sensors is their short lifespan when exposed to a moist environment.

Arduino MEGA: Arduino is open source electronic platform based on easy-to-use hardware and software. The board is equipped with sets of digital and analog input/output pins that may be interfaced to various expansion boards and other circuits. Since it is user friendly, this board is used.

MQ-135 Gas Sensor: The sensor senses the gases like ammonia, nitrogen, oxygen, Alcohol, sulphide and smoke. Urea contains ammonia and MQ135 is very sensitive to ammonia. It has potential to detect different gases. The operating voltage of this gas sensor is from $2.5 \mathrm{~V}$ to $5.0 \mathrm{~V}$. The conductivity of the gas sensor increases as the concentration of polluting gas increases and also this gas sensor is 
inexpensive.

LEDs: A Light-Emitting diode (LED) is a semiconductor device that emits light when an electric current is passes through it. Light is produced when the particles that carry the current combine together within the semiconductor material. There are three color LEDs used GREEN-used to indicate the milk is not adulterated and safe to drink, YELLOW-indicates that the milk is moderately adulterated but not much harmful to humans, RED-used to indicate the milk is highly adulterated and not at all safe to drink.

LCD: Liquid-Crystal display is a low-power, flat-panel display or electrically modulated optical device that uses the light modulating properties if liquid crystals combined with polarizers. Liquid crystals do not emit light directly, instead using a backlight or reflector to produce images in color or monochrome. LCD is used in many digital devices to display numbers or images. It uses a crystal to produce visible images. Here it is used to display the values indicated by the sensors.

The pictures given below represent the software and hardware components of our project.

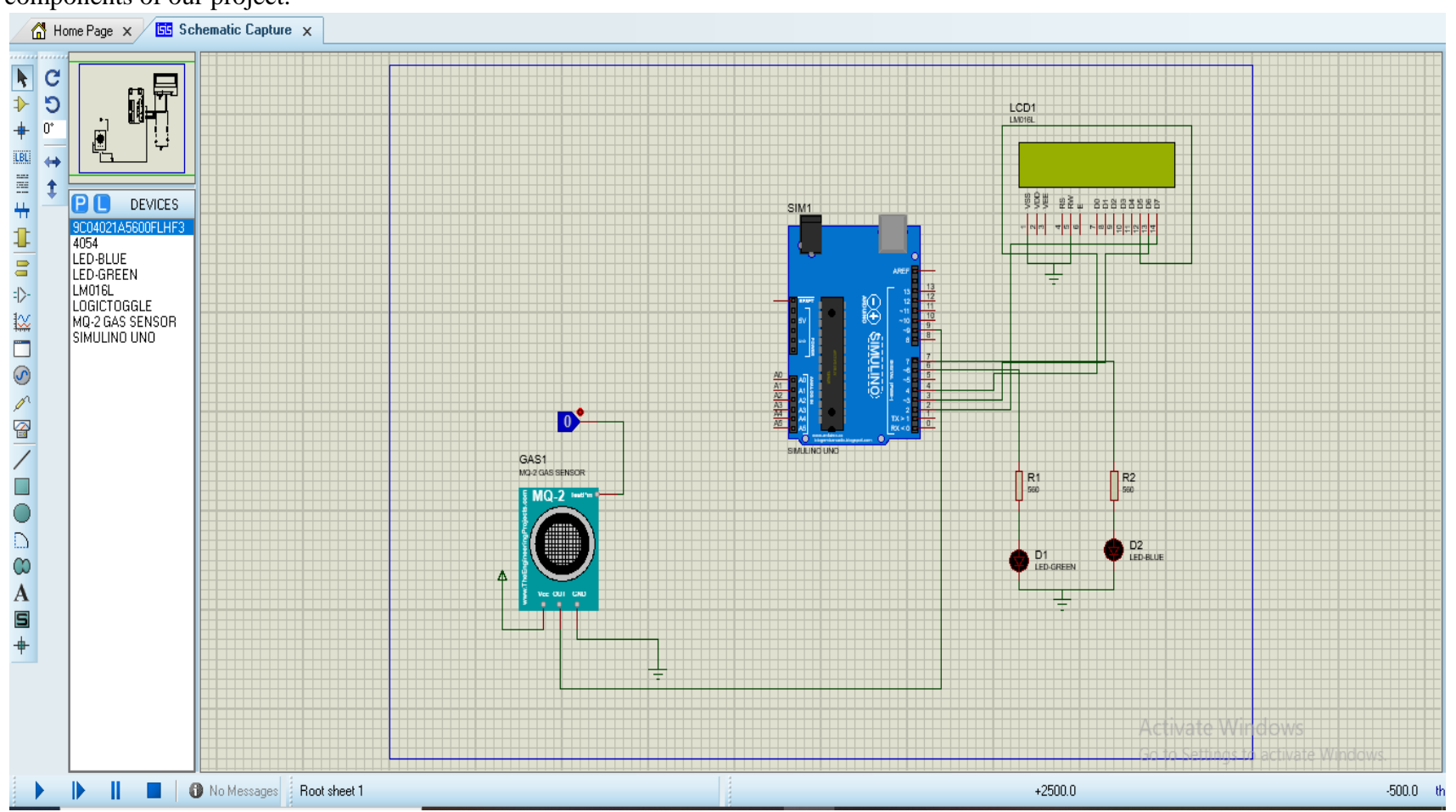

Fig 3: Simulation of Gas sensor

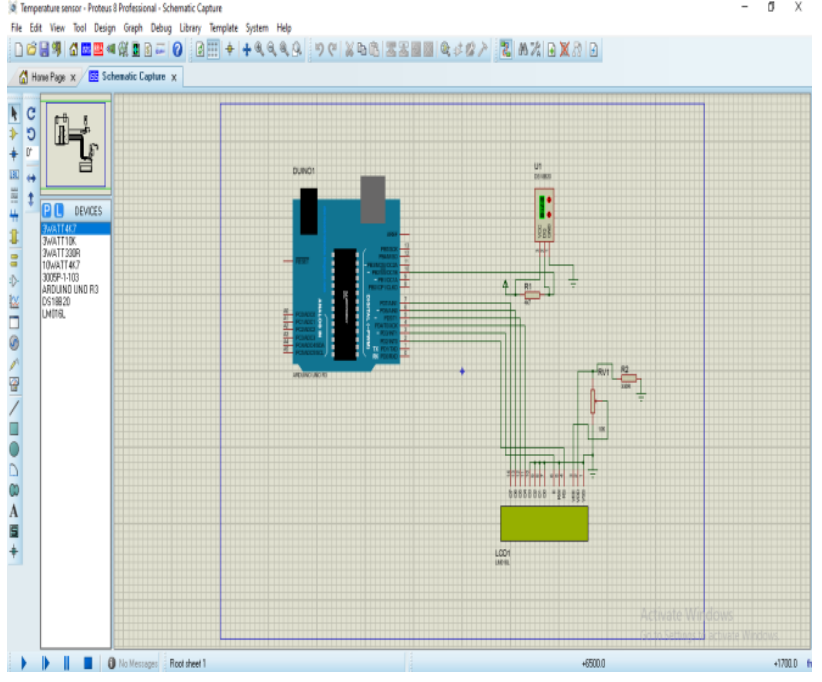

Fig 2: Simulation of Temperature sensor 


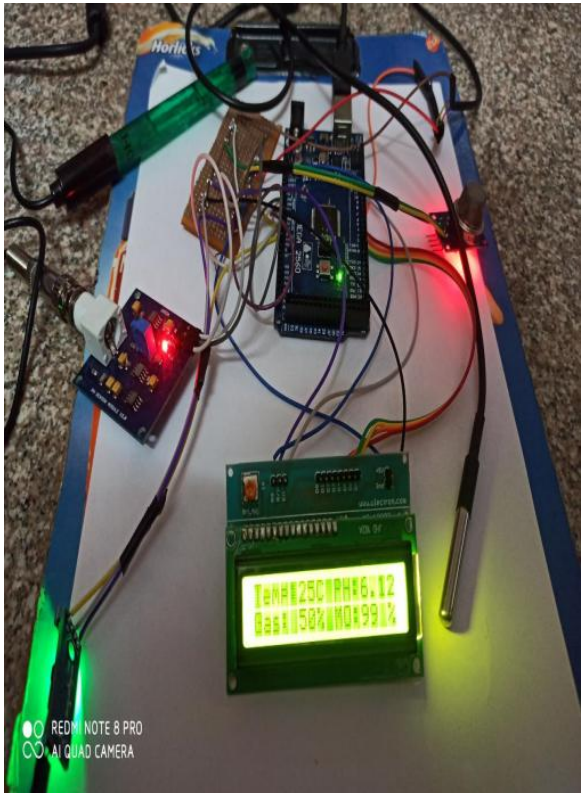

Fig 4: Hardware Connection

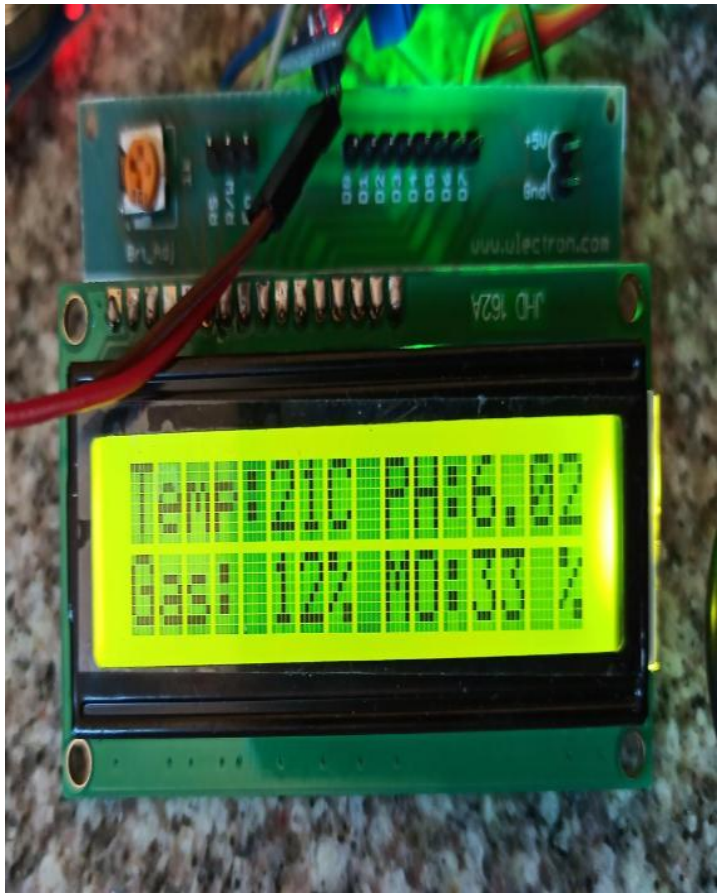

Fig 5: LCD displaying values of temperature, $\mathrm{pH}$, percentage of gas and dryness

Values obtained for pasteurized milk

\begin{tabular}{|l|l|l|l|l|}
\hline Parameters & $\mathrm{pH}$ & Temperature & Moisture & Gas \\
\hline $\begin{array}{l}\text { Values } \\
\text { obtained for } \\
\text { pasteurized } \\
\text { milk }\end{array}$ & 6.02 & 21 & $67 \%$ & $12 \%$ \\
\hline $\begin{array}{l}\text { Values } \\
\text { obtained for } \\
\text { spoilt milk }\end{array}$ & 4.64 & 19 & $72 \%$ & $15 \%$ \\
\hline
\end{tabular}

\section{CONCLUSION}

Adulteration in milk is normally present in the form of prohibited substances which are often added to milk or it is completely synthetic. This is usually resorted to for financial gain. Many a times carelessness and lack of hygienic conditions of processing, storing, transportation and marketing also lead to adulteration. Such types of adulteration are quite common in developing countries and backward countries. Adulteration of milk is a serious concern in developed countries also. In these countries advanced methods are adopted to adulterate milk and need sophisticated methods for detection. Consumers are either cheated or often become victim of diseases by consuming adulterated milk. Quality control tests for milk are a considerable aspect to assure adulterant free milk for consumption. Gastro enteritis, hypertension, renal diseases, skin, eye, heart problem and cancer are some of the common diseases caused by consuming adulterated milk. Detection techniques comprise of simple visual inspection to complex biological processes. This paper gives an overview of the different elements used to adulterate milk, the methodology to identify the adulterants and an elaborate study on electronic methods adopted to identify adulterants. It is expected that our device will help the researchers to get an overall idea of the electronic sensor based adulterant detection techniques.

\section{ACKNOWLEDGEMENTS}

Our thanks to the experts who have contributed towards development of this project

\section{REFERENCES}

[1] Saadia.H.H Shinwy, Adel M. El-Kholy and et.al, Detection Of Adulteration In Milk And Some Dairy Products Assiut Veterinary Medical Journal, Volume.64, Issue.157, 2018, 1-10.

[2] ManjotKaur and JiwanjotKaur, Dairy Products and their Adulteration, Journal of dairy Science and Technology, Volume7, Issue2, 2018, 11-14.

[3] Dr. G. Rajakumar, Dr. T.Ananth Kumar, Dr. T.S. Arun Samuel , Dr. E.Muthu Kumaran, Iot based milk monitoring system for detection of milk adulteration, International Journal of Pure and Applied Mathematics Volume 118 No. 9 2018, 21-32

[4] Jai Desai, Rohit Kadav, Bhakte Karki, Varsha Patil, Prof. Lalita Moharkar, Detection of Adulteration in Milk using Optical Sensor, Pramana Research Journal, ISSN NO: 2249-2976

[5] Dadasaheb Navale \&Shelley Gupta, Detection of an adulteration present in milk and milk products, International journal of latest technology in engineering, Management \& Applied Science (ULTEMAS) Volume V, issue VI, June 2016| ISSN: 2278-2540.

[6] Tanzina Azad \& Shoeb Ahmed, common milk adulteration and their detection techniques, International journal of food contamination 3, 22(2016), ISSN: 21962804.

[7] Y. G. Lee, H. Y. Wu, C. L. Hsu, C. J. Liang, H. D. Yuan."A Rapid And Selective Method For Monitoring The Growth Of Coliforms In Milk Using The Combination Of Amperometric Sensor And Reducing Of Methylene Blue," Sensors And Actuators B: Chemical, Vol. 141, no. 2, 2009, pp. 575-580.

[8] H. M. Al-Qadiri, M. Lin, M. A. Al-Holy, A. G. Cavinato 
and B. A. Rasco, "Monitoring Quality Loss of Pasteurized Skim Milk Using Visible And Short Wavelength Near-Infrared Spectroscopy And Multivariate Analysis," Journal of Dairy science, Vol. 91, no. 3, 2008, pp. 950- 958 .
[9] N. Nicolaou, Y. Xu and R. Goodacre, "Fourier Transform Infrared Spectroscopy And Multivariate Analysis For The Detection And Quantification Of Different Milk Species," Journal Of Dairy Science, Vol. 93, NO. 12, 2010, pp. 5651-5660. 